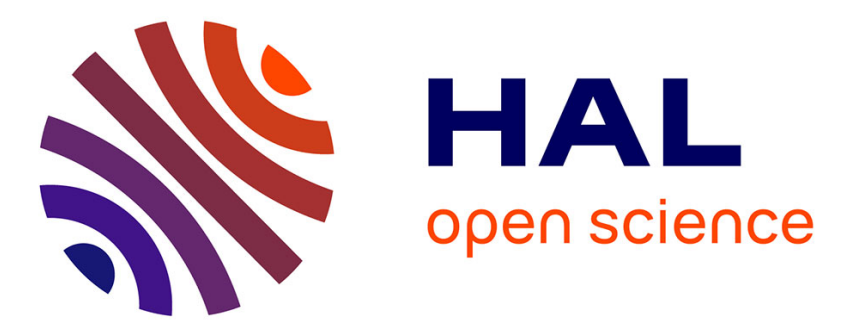

\title{
Dystonies monogéniques : l'hypothèse dopaminergique revisitée
}

Arnaud Blanchard, A. Roubertie, M. y Frédéric, M. Claustres, Gwenaëlle Collod-Béroud

\section{- To cite this version:}

Arnaud Blanchard, A. Roubertie, M. y Frédéric, M. Claustres, Gwenaëlle Collod-Béroud. Dystonies monogéniques : l'hypothèse dopaminergique revisitée. Revue Neurologique, 2010, 166 (4), pp.389-399. 10.1016/j.neurol.2009.09.007 . hal-01669962

\section{HAL Id: hal-01669962 https://hal.science/hal-01669962}

Submitted on 21 Dec 2017

HAL is a multi-disciplinary open access archive for the deposit and dissemination of scientific research documents, whether they are published or not. The documents may come from teaching and research institutions in France or abroad, or from public or private research centers.
L'archive ouverte pluridisciplinaire HAL, est destinée au dépôt et à la diffusion de documents scientifiques de niveau recherche, publiés ou non, émanant des établissements d'enseignement et de recherche français ou étrangers, des laboratoires publics ou privés. 


\title{
Revue générale
}

\section{Dystonies monogéniques : l’hypothèse dopaminergique revisitée}

\section{Monogenetic dystonia: Revisiting the dopaminergic hypothesis}

\author{
A. Blanchard ${ }^{a, b}$, A. Roubertie ${ }^{c}$, M.Y. Frédéric ${ }^{a, b}$, M. Claustres ${ }^{a, b, d}$, G. Collod-Béroud $^{a, *, b}$ \\ a Inserm, U827, institut universitaire de recherche clinique, 641, avenue du Doyen-Gaston-Giraud, 34093 Montpellier cedex 05, France \\ ${ }^{\mathrm{b}}$ UFR médecine, université de Montpellier-1, 34000 Montpellier, France \\ ${ }^{\mathrm{C}}$ Service de neuropédiatrie, hôpital Guy-de-Chauliac, CHU de Montpellier, 34000 Montpellier, France \\ ${ }^{\mathrm{d}}$ Service de génétique moléculaire, hôpital Arnaud-de-Villeneuve, CHU de Montpellier, 34000 Montpellier, France
}

I N F O A R T I C L E

Mots clés :

Dystonies

Ganglions de la base

Physiopathologie

Dopamine

Hétérogénéité génétique

\section{Keywords:}

Dystonia

Basal ganglia

Physiopathology

Dopamine

Genetic heterogeneity

\begin{abstract}
R É S U M É
Les dystonies sont des maladies du mouvement cliniquement et génétiquement hétérogènes dont le champ d'investigation est en plein essor. L'identification récente du gène THAP1, impliqué dans la forme primaire DYT6, relance la très intéressante question de l'implication in fine de la voie de la dopamine dans les différentes formes de dystonies. L'objectif de cette revue est de reprendre les différents travaux publiés ces dernières années afin d'évaluer cette hypothèse, mais également de mettre en avant toute la difficulté d'identifier un schéma global de régulation dans ces maladies que l'on commence à peine à déchiffrer.
\end{abstract}

\begin{abstract}
A B S T R A C T
Dystonias are clinically and genetically heterogeneous neurological disorders that affect movement, and are the focus of much investigative work. The recent identification of mutations in the gene THAP1 in DYT6 dystonia reopens the very interesting question of the in fine involvement of dopamine in the different types of dystonia. In this review, we will go through the recent literature in order to evaluate the many contributions to this theory as well as to highlight the difficulties in identifying a global regulatory pathway for the different forms of this disease that we are just starting to decipher.
\end{abstract}

Le terme de "dystonie " est souvent source de confusions puisqu'il correspond à la fois à un symptôme et au nom d'un groupe de maladies, appelées dystonies. Le symptôme, ou la manifestation clinique, correspond à une anomalie de contrôle du mouvement avec des contractions musculaires involontaires qui entraînent des mouvements de torsion et des postures anormales. Il s'agit du troisième mouvement anormal en fréquence chez l'homme après le parkinson et le

\footnotetext{
* Auteur correspondant.

Adresse e-mail : gwenaelle.collod-beroud@inserm.fr (G. Collod-Béroud).
} 
tremblement. La dystonie en tant que maladie s'intègre dans différents syndromes qui peuvent être schématiquement classés en deux types : les syndromes primaires et les secondaires. Les syndromes dystoniques sont dits " primaires " lorsqu'aucune cause exogène n'est identifiée (traumatisme, anoxie péri- ou postnatale), lorsqu'il n'y a aucun argument en faveur d'une maladie métabolique ou neurodégénérative ; un tremblement ou des myoclonies sont parfois associés, mais il n'y a pas d'autre signe neurologique (en particulier, il n'y a pas de détérioration mentale). Une grande partie de ces formes de dystonies primaires a une composante héréditaire. On distingue les dystonies avec un âge d'apparition précoce qui ont tendance à progresser vers des dystonies plus sévères, des dystonies avec un âge d'apparition plus tardif qui restent généralement focales comme les dystonies cervicales, les dystonies de fonction (crampe de l'écrivain, du violoniste...), les dystonies oromandibulaires et les blépharospasmes. Les dystonies " secondaires" comprennent des syndromes dans lesquels la dystonie est associée à un ensemble d'autres signes, ou est le résultat d'une lésion cérébrale. Ce groupe inclut les syndromes dystoniques-plus, correspondant à des entités cliniquement bien définies et génétiquement déterminées (dystonies dopa-sensibles [DRD], dystonie myoclonique et syndromes dystonie-parkinsonisme de début précoce qui feront partie de notre propos). Par ailleurs, plus de 42 maladies héréditaires associées à une neurodégénérescence peuvent être associées à des manifestations dystoniques (Geyer et Bressman, 2006).

La physiopathologie des dystonies reste encore largement inconnue. L'étude des dystonies secondaires symptomatiques (i.e. résultant d'une lésion cérébrale focale), formes dans lesquelles la région du cerveau affectée peut être identifiée, montre que les anomalies structurales ou biochimiques sont retrouvées majoritairement dans les ganglions de la base (striatum constitué du noyau caudé et du putamen, globus pallidus, noyau subthalamique et substance noire) ou le thalamus, qui sont impliqués dans le contrôle central du mouvement, mais également au niveau du lobe pariétal, du cervelet et du tronc cérébral (Geyer et Bressman, 2006). Les ganglions de la base sont, en effet, au centre d'une boucle de régulation du mouvement dont la voie d'entrée est le striatum (Fig. 1). Celui-ci reçoit des projections corticales excitatrices et renseigne à son tour le globus pallidus interne (GPi) par deux voies :

- une voie directe (trans-striatale) qui est constituée par des neurones striataux GABAergiques (inhibiteurs) exprimant des récepteurs D1 à la dopamine et projetant sur le GPi et la substance noire réticulée $(\mathrm{SNr})$;

- la voie indirecte (striato-pallido-subthalamique) qui aboutit aussi au niveau du GPi/SNr en partant du striatum.

Celui-ci projette sur le globus pallidus externe (GPe) via des neurones GABAergiques exprimant des récepteurs D2 à la dopamine. Le GPe inhibe alors le noyau subthalamique (NST) dont les neurones glutamatergiques excitent le GPi et la SNr. Dans les deux voies, GPi et SNr ont ensuite une action inhibitrice sur le thalamus, stimulateur du cortex moteur et prémoteur (Fig. 1). Les ganglions de la base, via la voie directe,

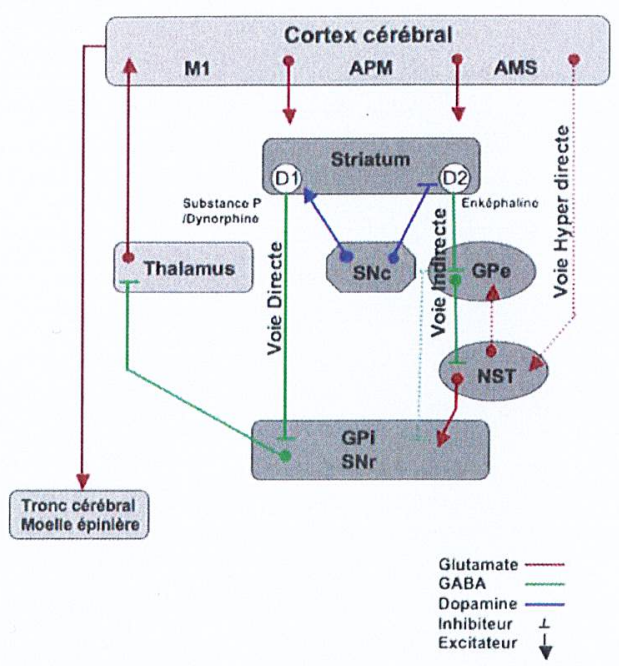

Fig. 1 - Organisation fonctionnelle des ganglions de la base (AMS : aire motrice supplémentaire ; APM : aire prémotrice ; M1 : cortex moteur primaire ; GPe : globus pallidus externe; GPi : globus pallidus interne; NST : noyau subthalamique; $\mathrm{SNr}$ : substance noire pars reticula ; SNc : substance noire pars compacta).

Functional organization of the basal ganglia (AMS: supplementary motor area; APM: premotor cortex; M1: primary motor cortex; GPe: globus pallidus, external segment; GPi: globus pallidus, internal segment; NST: subthalamic nucleus; SNr: substantia nigra pars reticulata; SNc: substantia nigra pars compacta).

sont classiquement impliqués dans la planification et l'exécution d'un mouvement, spécialement ceux qui ont été appris et pour lesquels un programme moteur a été mis en place, la voie indirecte permettant de supprimer tout mouvement parasite similaire à celui désiré. Placée au centre de ces deux voies, la substance noire compacte $(\mathrm{SNc})$ module l'activité du striatum au moyen de fibres dopaminergiques qui pourront être interprétées de façon opposée suivant le type de récepteurs que possède la structure receveuse (ici le striatum). Les récepteurs $\mathrm{D} 1$ exprimés par les neurones striataux de la voie directe médieront une dépolarisation du neurone postsynaptique contrairement aux récepteurs D2 exprimés par les neurones striataux de la voie indirecte qui en provoqueront une hyperpolaristion.

Chef d'orchestre d'un ballet complexe, la dopamine est donc au cœur de la régulation du mouvement exercée par ces ganglions et son rôle dans la physiopathologie de certaines dystonies évoqué (Vidailhet et al., 1999 ; Perlmutter et Mink, 2004 ; Vitek, 2002 ; Wichmann, 2008 ;Vidailhet et al., 2009). L'hypothèse de cette implication est soutenue par les nouvelles techniques d'imagerie, en particulier la tomographie d'émission de positrons (TEP) et la tomographie par émission de simples photons (TESP). Une diminution de la liaison aux récepteurs $\mathrm{D} 2$ de la dopamine dans le striatum a été mise en évidence par TEP chez des patients présentant des dystonies focales (crampe de l'écrivain, blépharospasme et dystonie oromandibulaire) (Perlmutter et al., 1997; Horie et al., 2009), résultats confirmés dans la dystonie de type DYT1 
(Asanuma et al., 2005) et par TESP dans des dystonies myocloniques (Beukers et al., 2009), dystonies que nous aborderons dans le détail par la suite. Des résultats opposés ont, en revanche, été obtenus par TEP chez des patients présentant une dystonie dopasensible avec un accroissement de la disponibilité des récepteurs D2 (Rinne et al., 2004) ou une disponibilité normale des récepteurs dans un cas de dystonieparkinsonisme à évolution rapide (Zanotti-Fregonara et al., 2008).

Des anomalies de signal ont été mises en évidence grâce aux IRM réalisées chez certains patients atteints de DYT1 au niveau du putamen et des globes pâles (Gavarini et al., 2008). Néanmoins, la signification de ces anomalies est encore inconnue et l'absence apparente de neurodégénérescence est une notion classique concernant les dystonies primaires, permettant de les classer dans la catégorie des "maladies neurofonctionnelles ". Il s'agit de maladies liées à des anomalies, visibles à l'échelle microscopique, concernant la connectivité, la plasticité et/ou la régulation de la synapse.

Les dystonies sont classées selon trois critères :

- la topographie des signes cliniques observés ;

- l'âge d'apparition des symptômes ;

- l'étiologie de la maladie (primaire/secondaire).

À l'heure actuelle, 19 formes de dystonies monogéniques sont caractérisées et nommées de DYT1 à DYT20 (Tableau 1) (Ichinose et al., 1994 ; Lüdecke et al., 1995 ; Ozelius et al., 1997 ; Friedman et al., 2006; Zimprich et al., 2001; Makino et al., 2007; de Carvalho Aguiar et al., 2004 ; Rainier et al., 2004 ; Camargos et al., 2008 ; Weber et al., 2008 ; Fuchs et al., 2009 ; Breakefield et al., 2008). En effet, la forme DYT14 (Grotzsch et al., 2002) a finalement été révélée comme étant une dystonie DYT5 dopasensible associée à une grande délétion du gène guanosine triphosphate cyclohydrolase 1 (GCH1) (Wider et al., 2008). De nombreuses avancées ont été réalisées récemment avec la description de deux nouvelles formes : la forme DYT16, qui correspond à une dystonie autosomique récessive de début juvénile associée à des signes parkinsoniens (Camargos et al., 2008) ainsi que la forme DYT17 qui correspond à une forme autosomique récessive de dystonie focale dont le gène, encore inconnu, a été localisé sur le chromosome 20 (Chouery et al., 2008). Enfin, la base moléculaire de la forme DYT6 a été élucidée avec la découverte de mutations dans le gène THAP domain containing, apoptosis associated protein 1 (THAP1) (Fuchs et al., 2009). Le défaut moléculaire est maintenant décrypté dans neuf formes de dystonies et les fonctions des gènes identifiés sont très diverses, allant d'enzymes impliquées dans la synthèse de dopamine à des facteurs de transcription.

\section{DYT5 et le groupe des dystonies dopa- sensibles : premières implications de la dopamine}

Les DRD représentent moins de $5 \%$ des dystonies de l'enfant. La forme classique ou dystonie héréditaire progressive à fluctuations diurnes (DYT5) débute durant la première décade. La dystonie est plutôt posturale, au niveau des membres inférieurs (varus équin, marche digitigrade, douleur à la marche). Les fluctuations diurnes avec aggravation des manifestations en fin de journées, très évocatrices du diagnostic, sont observées chez les trois quarts des patients.

\begin{tabular}{|c|c|c|c|}
\hline Type de dystonie & $\begin{array}{c}\text { Mode de } \\
\text { transmission }\end{array}$ & $\begin{array}{l}\text { Localisation } \\
\text { chromosomique }\end{array}$ & Gène \\
\hline DYT1 : dystonie de torsion précoce & $A D$ & $9 q 34$ & TOR1A (Ozelius et al., 1997) \\
\hline DYT2 : dystonie de torsion autosomique récessive & $\mathrm{AR}$ & $?$ & ? \\
\hline DYT3 : dystonie-parkinsonisme (Lubag) & $\mathrm{XR}$ & $\mathrm{Xq13.1}$ & TAF1 (Makino et al., 2007) \\
\hline DYT4 : dystonie laryngée & $\mathrm{AD}$ & $?$ & $?$ \\
\hline \multirow[t]{3}{*}{ DYT5 : dystonie dopasensible } & $\mathrm{AD}$ & $14 \mathrm{q} 22.1-\mathrm{q} 22.2$ & GCH1 (Ichinose et al., 1994) \\
\hline & AR & $11 \mathrm{p} 15.5$ & TH (Lüdecke et al., 1995) \\
\hline & AR & 2p14-p12 & SPR (Friedman et al., 2006) \\
\hline DYT6 : dystonie mixte débutant chez le jeune adulte & $\mathrm{AD}$ & $8 p 21-22$ & THAP1 (Fuchs et al., 2009) \\
\hline DYT7 : dystonie focale débutant à l'âge adulte & $\mathrm{AD}$ & $18 \mathrm{p}$ & $?$ \\
\hline DYT8 : dyskinésie paroxystique non kinésigénique & $\mathrm{AD}$ & $2 q 33-q 35$ & MR-1 (Rainier et al., 2004) \\
\hline DYT9 : choréoathétose paroxystique & $\mathrm{AD}$ & $1 \mathrm{p}$ & $?$ \\
\hline DYT10 : dyskinésie paroxystique kinésigénique & $\mathrm{AD}$ & $16 \mathrm{p} 11.2-\mathrm{q} 12.1$ & $?$ \\
\hline DYT11 : dystonie myoclonique & $\mathrm{AD}$ & $7 q 21$ & SGCE (Zimprich et al., 2001) \\
\hline DYT12 : dystonie-parkinsonisme à évolution rapide & $\mathrm{AD}$ & $19 q 13$ & $\begin{array}{l}\text { ATP1A3 (de Carvalho Aguiar } \\
\text { et al., 2004) }\end{array}$ \\
\hline DYT13 : dystonie multifocale/segmentaire & $\mathrm{AD}$ & $1 \mathrm{p} 36.13-36.32$ & $?$ \\
\hline \multicolumn{4}{|l|}{ DYT14 = DYT5 } \\
\hline DYT15 : dystonie myoclonique & $\mathrm{AD}$ & 18 p11 & $?$ \\
\hline DYT16 : dystonie-parkinsonisme à début précoce & AR & $2 q 31.2$ & PRKRA (Camargos et al., 2008) \\
\hline DYT17 : dystonie focale de torsion & AR & $20 \mathrm{p} 11.22-\mathrm{q} 13.12$ & $?$ \\
\hline DYT18 : dyskinésie paroxystique induite par l'exercice & $\mathrm{AD}$ & $1 \mathrm{p} 35-\mathrm{p} 31.3$ & SLC2A1 (Weber et al., 2008) \\
\hline DYT19 : dyskinésie paroxystique kinésigénique (DPK2) & $\mathrm{AD}$ & $16 q 13-q 22.1$ & $?$ \\
\hline DYT20 : dyskinésie paroxystique non kinésigénique (DPNK2) & $\mathrm{AD}$ & $2 q 31$ & $?$ \\
\hline
\end{tabular}


En l'absence de traitement, la dystonie peut se généraliser. Les signes parkinsoniens (akinésie, rigidité) apparaissent plus tard au cours de l'évolution, ou peuvent être le symptôme inaugural de la maladie lorsque les premiers signes apparaissent chez les adultes. Les symptômes dystoniques sont améliorés de façon spectaculaire grâce à de faibles doses de Ldopa (Breakefield et al., 2008). Il existe d'autres formes de dystonies de l'enfant sensibles à la L-dopa ou aux agonistes dopaminergiques. Cependant, l'efficacité du traitement est souvent moins spectaculaire que chez les patients atteints de dystonie DYT5. Les tableaux cliniques sont variés et souvent atypiques (tableau limité à des contractures musculaires d'effort, tableau de paraplégie spastique, tableau de début infantile avec retard précoce de développement avec signes moteurs [akinésie, hypotonie, crises oculogyres et à dyskinésies buccofaciales]) et signes dysautonomiques liés au déficit en cathécholamines (ptosis, myosis, troubles de la régulation thermique). L'existence de fluctuations diurnes est très inconstante, mais peut orienter le diagnostic.

Dans la dystonie DYT5 et les autres formes de DRD, la dopamine et son défaut de synthèse sont clairement au cœur de la problématique. La forme " classique " de dystonie dopasensible est transmise sur un mode autosomique dominant à pénétrance incomplète et expressivité variable. Elle est liée à des mutations du gène GCH1 impliqué dans la synthèse des bioptérines, cofacteurs de la biosynthèse des monoamines cérébrales (Fig. 2) (Hyland et al., 2003). Près d'une centaine de mutations dans le gène CGH1 sont connues (Ichinose et al., 1994 ; Furukawa, 2004). Les autres formes de DRD sont autosomiques récessives, les mécanismes moléculaires impliquant les enzymes de la voie de la biosynthèse des monoamines cérébrales (déficit en CGH1 lié à des mutations récessives, déficit en tyrosine hydroxylase ou en sépiaptérine réductase notamment, une dizaine d'enzymes pouvant être impliquées) (Fig. 2) (Hyland et al., 2003 ; Pons, 2009 ; Clot et al., 2009). Le dosage des ptérines et l'analyse du profil des métabolites des amines cérébrales du liquide céphalorachidien orientent vers le niveau du blocage enzymatique.

\section{DYT1 et protéine chaperon : une fonction inattendue}

La dystonie DYT1, de transmission autosomique dominante, est la forme la plus fréquente des dystonies primaires débutant dans l'enfance ( $90 \%$ des cas dans la population juive Ashkénaze, $60 \%$ dans la population non juive). En France, une étude récente a estimé la fréquence de la mutation à $0,17 / 100000$ (Frédéric et al., 2008). L'âge moyen de début est de 12,5 ans, au niveau d'un membre dans $94 \%$ des cas (posture anormale du pied à la marche, difficultés à l'écriture : main crispée sur le stylo, crampes à l'écriture) (Bressman et al., 2000). La dystonie peut s'étendre à d'autres parties du corps, devenant multifocale ou généralisée chez environ $70 \%$ des enfants, en cinq à dix ans. Le handicap fonctionnel peut être sévère et est lié à la topographie de l'atteinte durant ces premières années et aux complications orthopédiques, la progression étant ensuite beaucoup plus lente. Il existe cependant une grande variabilité phénotypique interindividuelle et intrafamiliale, l'atteinte pouvant rester strictement

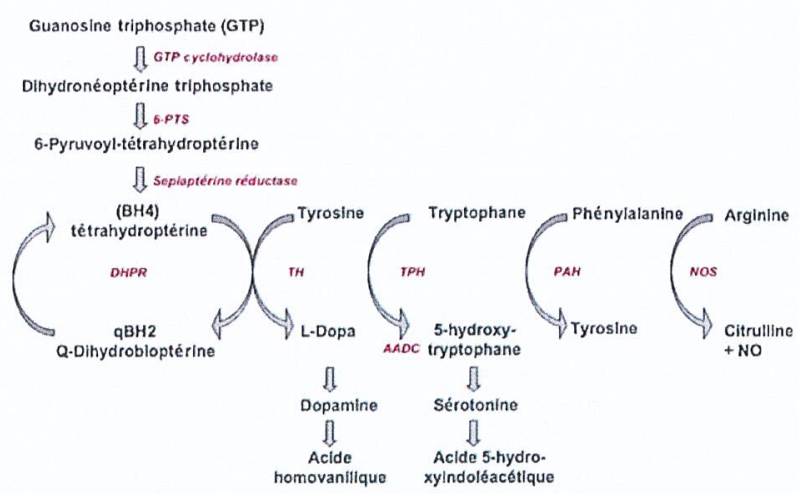

Fig. 2 - Voie de synthèse des tétrahydroptérines menant à la synthèse de la dopamine (6-PTS : 6-pyruvoyl-tétrahydroptérine synthase; DHPR : dihydroptérine réductase ; TH : tyrosine hydroxylase ; TPH : tryptophane hydroxylase ; PAH : phénylalanine hydroxylase ; NOS : nitric oxyde synthase ; AADC : décarboxylase de $\mathrm{L}$-acide amine) (adapté de Hyland et al., 2003). Involvement of tetrahydrobiopterin in dopamine metabolism (adapted from Hyland et al., 2003) (6-PTS: 6-pyruvoyl tetrahydropterin synthase; DHPR: dihydropteridine reductase; TH: tyrosine hydroxylase; TPH: tryptophan hydroxylase; PAH: phenylalanine hydroxylase; NOS: nitric oxide synthase; AADC: aromatic L-amino acid decarboxylase).

focale. La pénétrance est par ailleurs incomplète. Les traitements pharmacologiques ont une efficacité souvent limitée. Des cas ponctuels de stabilisation ou d'amélioration sous anticholinergique, dopathérapie, ou dépléteur en dopamine ont été rapportés (Jankovic, 2006).

Cette forme de dystonie est liée à une mutation du gène TOR1A qui code la torsineA, un membre de la super famille des ATPases $\mathrm{AAA}^{+}$(ATPases associated with a variety of cellular activities) (Ozelius et al., 1997). La fonction de cette protéine est, et a été, très étudiée et tout un catalogue de fonctions a été mis en évidence. Deux ressortent principalement de ces études : la fonction de "protéine chaperon " (Morange, 2000), i.e. d'une protéine empêchant toutes liaisons non souhaitées, qu'elles soient physiologiques (aide dans le repliement de protéines ou désassemblage de complexes protéiques) ou non (dégradation d'agrégats causés par un stress) (Torres et al., 2004 ; Cao et al., 2005 ; Hewett et al., 2007 ; Granata et al., 2008), ainsi qu'un rôle plus « structural » dans l'organisation de l'enveloppe nucléaire et des compartiments du réticulum endoplasmique (RE) (Naismith et al., 2004 ; Goodchild et al., 2005).

Dès 2000, l'implication de la dopamine dans ces formes sévères semble se dessiner avec l'observation d'une réduction du niveau de dopamine dans le putamen et le noyau caudé chez un patient DYT1 (Furukawa et al., 2000). Suivront les démonstrations d'une augmentation de la demi-vie de la dopamine (Augood et al., 2002) ainsi que d'une augmentation de taille des corps cellulaires dopaminergiques dans la substance noire (Rostasy et al., 2003). En 2007, cette hypothèse est reprise dans un article de Pisani et al., 2007 qui replace également le striatum au centre du débat. Ce dernier se 
compose à près de $95 \%$ de neurones GABAergiques, les $5 \%$ restant correspondant à quatre types d'interneurones dont un type seulement est cholinergique (Zhou et al., 2002). Ces interneurones cholinergiques ont une activité synaptique autonome (neurones toniquement actifs) et agissent comme une sorte de pacemaker. Leur activité basale est régulée, d'une part, par le fort taux d'acétylcholinestérase dans le striatum qui assure la dégradation de l'acétylcholine (Ach) dans la fente intersynaptique (Zhou et al., 2002) et, d'autre part, par un rétrocontrôle négatif sur la libération de l'Ach médié par les autorécepteurs muscariniques M2/M4 (Calabresi et al., 1998). Des analyses plus récentes démontrent que l'activité de ces interneurones et la libération de l'Ach sont également modulées par la dopamine via l'action conjointe et antagoniste des récepteurs D2 qui permettent, d'une part, de réduire les décharges (Deng et al., 2007) et, d'autre part, des récepteurs D5 qui augmentent l'excitabilité des interneurones (Aosaki et al., 1998). La torsineA, bien qu'ubiquitaire, montre une expression plus élevée dans certains neurones comme les innervations dopaminergiques et les interneurones cholinergiques des ganglions de la base (Augood et al., 1999 ; Shashidharan et al., 2000). Dans un modèle de souris surexprimant la torsineA mutée (Sharma et al., 2005), une anomalie de couplage des récepteurs D2 est observée, bien que le lien précis entre récepteurs $D 2$ et torsineA ne soit pas encore élucidé. L'activation des récepteurs D2 chez ces souris, au lieu de réduire la décharge des interneurones, va l'augmenter. Cette différence apparaît être le résultat d'un couplage inhibiteur exacerbé entre les récepteurs D2 et des canaux calvéoline Calv2 calciques qui régulent l'ouverture $\mathrm{Ca}^{2+}$ dépendante des canaux potassiques Kir3 après la décharge (Pisani et al., 2006). Ce mécanisme qui n'est pas encore bien déchiffré semble passer par RGS9 qui est un régulateur des récepteurs D2 couplés aux canaux Cav2 dans les interneurones (Cabrera-Vera et al., 2004). L'augmentation des décharges cholinergiques des interneurones pourrait provoquer une libération plus importante d'Ach expliquant l'efficacité des substances anti-cholinergiques dans le traitement des dystonies.

Plus récemment, dans ce même modèle de souris, une augmentation anormale de l'activité synaptique GABAergique a été mise en évidence (Sciamanna et al., 2009). Cette dérégulation pourrait être en partie due à un dysfonctionnement des récepteurs $\mathrm{D} 2$.

La torsineA pourrait également intervenir tout simplement en tant que protéine chaperon fortement impliquée dans l'élimination des protéines mal repliées induites par le fort taux d'espèces réactives à l'oxygène produites au cours de la synthèse de la dopamine (Cohen, 1984) comme cela a été suggéré dans la maladie de Parkinson (Dauer et Przedborski, 2003).

\section{DYT11 et l'\&-sarcoglycane : une question de structure membranaire?}

La forme DYT11 de dystonie débute durant la première ou seconde décade ; elle se caractérise par des myoclonies affectant principalement les muscles proximaux (membres supérieurs, cou, tronc) et des mouvements dystoniques le plus souvent focaux, impliquant la partie supérieure du corps (dystonie cervicale ou des membres supérieurs). Ces manifestations sont isolées ou associées avec une sévérité variable. Les myoclonies sont souvent améliorées par la prise d'alcool. Chez certains patients, la présence de troubles psychiatriques tels que des crises de panique et des troubles obsessionnels compulsifs a été rapportée (Maréchal et al., 2003 ; Hess et al., 2007). En 2001, une mutation dans le gène codant pour l' $\varepsilon$ sarcoglycane (SGCE) (Zimprich et al., 2001) a été identifiée dans cing familles présentant une forme de dystonie associée à des myoclonies. Le gène SGCE est un gène soumis à empreinte maternelle (la copie mutée est transmise par le père, la copie maternelle est inactivée) (Grabowski et al., 2003). L'étude récente de plusieurs mutations faux-sens (un acide aminé remplacé par un autre acide aminé) a révélé que ces protéines mutantes ne sont pas normalement adressées à la membrane où elles doivent remplir leur fonction. Elles sont retenues au niveau intracellulaire, ubiquitinylées et dégradées par le protéasome (Esapa et al., 2007). La torsineA, en tant que protéine chaperon, serait un partenaire actif dans ce processus d'élimination (Esapa et al., 2007).

L'E-sarcoglycane appartient à la famille des sarcoglycanes qui est bien connue pour son rôle dans le muscle où elle forme un complexe avec la dystrophine pour relier le cytosquelette à la matrice extracellulaire. Quatre membres de cette famille $(\alpha$, $\beta, \gamma$ et $\delta)$ sont exprimés principalement dans le muscle et des anomalies de ces gènes ont été rapportées dans des formes autosomiques récessives de dystrophies musculaires des ceintures (Angelini et al., 1999). Peu de données anatomopathologiques sont disponibles pour les membres de cette famille dans le cerveau (Waite et al., 2009). La forme $\varepsilon$ sarcoglycane ainsi que la forme $\zeta$ récemment identifiée sont retrouvées dans différentes régions cérébrales (Wheeler et al., 2002 ; Shiga et al., 2006) tandis que plusieurs isoformes de la dystrophine sont exprimées (Dp71, Dp140 et Dp260) (Blake et al., 2002). Le complexe sarcoglycane, s'il existe dans le cerveau, est donc significativement différent du complexe musculaire. Son éventuelle implication dans une régulation de la structure de la membrane en association avec la dystrophine n'est pas connue. Le gène SGCE, quant à lui, est fortement exprimé dans les neurones et notamment les neurones dopaminergiques (Xiao et LeDoux, 2003 ; Nishiyama et al., 2004 ; Chan et al., 2005).

Dans un modèle animal de souris avec absence de Sgce par knock-out, les souris présentent similairement des myoclonies, un déficit moteur, une hyperactivité et des signes d'anxiété et de dépression. De façon intéressante, un taux anormalement haut de dopamine est observé dans le striatum (Yokoi et al., 2006), montrant à nouveau une dérégulation de la voie dopaminergique dans les dystonies myocloniques et impliquant un lien entre dopamine et $\varepsilon$-sarcoglycane. De par la fonction "structurale » de la famille des sarcoglycanes dans le muscle, l'hypothèse d'une dérégulation de la structure membranaire provoquée par des modifications subtiles au niveau de l'architecture neuronale peut être proposée. Cette dérégulation de la membrane pourrait provoquer une exacerbation de la libération de dopamine ou une perturbation de sa recapture et/ou de sa dégradation. Il faut noter cependant que chez deux patients présentant une dystonie myoclonique liée à une délétion partielle du gène SGCE, le bénéfice d'un 
traitement par la L-dopa a été rapporté (Luciano et al., 2009) et que ces résultats sont en contradiction avec les données expérimentales obtenues chez la souris.

Enfin, la possibilité de l'implication d'autres gènes dans cette forme de dystonie associée à des myoclonies a été mise en évidence dans une étude italienne (forme DYT15) (Valente et al., 2003)

\section{DYT3, dystonie-parkinsonisme lié à l'X : une régulation des récepteurs dopaminergiques ?}

La dystonie de Lubag est une dystonie-parkinsonisme qui se caractérise par une dystonie sévère et progressive dans les 1015 premières années, associée ou remplacée par un syndrome parkinsonien dans les années suivantes. Il s'agit d'un cas particulier, puisqu'il correspond au seul cas de dystonie monogénique pure avec une dégénérescence de neurones rapportée dans le noyau caudé et le putamen (Goto et al., 2005). Cette forme de dystonie est causée par une mutation unique transmise chez les mâles principalement, par un ancêtre commun de génération en génération sur l'île de Panay aux Philippines (effet fondateur) (Lee et al., 1976 ; Kupke et al., 1990). Ce syndrome dystonique est lié à l'insertion d'un rétrotransposon dans le gène TATA-binding protein-associated factor 1 (TAF1) (Makino et al., 2007). La localisation de TAF1 sur le chromosome $\mathrm{X}$ permet de comprendre le mode de transmission de cette pathologie. La majorité des personnes atteintes correspond à des mâles chez qui le gène TAF1 est porté par l'unique copie du chromosome X. Les quelques femmes atteintes correspondent aux cas où la copie "saine " du gène TAF1 a été réduite au silence dans le cadre de l'inactivation aléatoire d'un des chromosomes $\mathrm{X}$. Ce gène code pour un composant essentiel du complexe TFIID qui appartient à la voie de transcription de gènes médiée par la RNA polymérase II (Hisatake et al., 1993). Il régule ainsi un grand nombre de gènes impliqués dans la division cellulaire et la prolifération. Chez les patients, une diminution de l'expression du gène DRD2 codant pour les récepteurs D2 est également observée (Makino et al., 2007). L'hypothèse d'une régulation du gène $\mathrm{DRD} 2$ par le gène TAF1 a été proposée par similarité à la régulation du gène $\mathrm{DRD} 2$ par le complexe $\mathrm{Sp} 1 /$ TAF4 qui est observée dans le contexte de la chorée d'Huntington (Dunah et al., 2002).

L'équipe de Goto et al. (2005), grâce à l'étude postmortem de cerveaux de ces patients, a permis d'éprouver une hypothèse de fonctionnement des ganglions de la base émise par Graybiel et al. (2000) avec une implication d'une troisième voie de régulation des ganglions de la base. Dans ce modèle, le striatum est partagé en deux parties fonctionnelles (Fig. 1) :

- la matrice d'où naissent les voies directe et indirecte telles que précédemment décrites ;

- les striosomes qui projettent sur la SNc des fibres inhibitrices GABAergiques (constituant la troisième voie) permettant la régulation ses décharges dopaminergiques.

Cette voie permettrait selon les auteurs d'expliquer l'évolution du tableau clinique d'un syndrome hyperkinétique (dystonie) à un syndrome hypokinétique (parkinson). Le premier stade correspondrait à la levée de l'inhibition striosomale due à sa neurodégénérescence. Il en résulterait une surstimulation dopaminergique de la SNc sur la matrice striatale et se traduirait sur le plan clinique par un phénotype hyperkinétique. Puis, la dégénérescence gagnant la matrice, il y aurait une perte des projections inhibitrices de la voie directe sur l'ensemble GPi/SNr et des projections de la matrice sur le GPe (première connexion de la voie indirecte). Une surinhibition du Gpi/SNr sur le thalamus provoquerait alors un syndrome hypokinétique reproduisant sur le plan clinique une dégénérescence de la SNc caractéristique de la maladie de Parkinson.

\section{DYT8 et dystonies intermittentes : seulement une question de toxicité ?}

Les dystonies paroxystiques non kinésigéniques (DPNK) sont des dystonies qui se présentent sous formes d'épisodes de mouvements anormaux de quelques minutes jusqu'à quelques heures et qui surviennent spontanément ou en réponse à des facteurs déclencheurs autres que des mouvements tels que café, exercice, colère, alcool ou fatigue (MIM\#118800). Des mutations du gène myofibrillogenesis regulator 1 (MR-1) ont été mises en évidence chez certains patients présentant une DPNK (Lee et al., 2004 ; Li et al., 2004 ; Rainier et al., 2004). Ce gène présente une forte homologie avec la glyoxylase II (HAGH). Cette enzyme participe à la conversion du méthylglyoxal, connu pour son activité mutagène et carcinogène et sa toxicité pour les neurones, en acide lactique. Le methylglyoxal est trouvé en grande quantité dans le café, le thé et le vin ou successivement en réponse à un stress. Le gène MR-1 a trois isoformes (Lee et al., 2004) et MR-1L, l'isoforme spécifique du cerveau, est une protéine qui est importée dans la mitochondrie grâce à une séquence d'adressage de 39 acides aminés (Ghezzi et al., 2009). Cette séquence, qui contient les trois mutations décrites à ce jour (Rainier et al., 2004; Ghezzi et al., 2009), est clivée avant l'insertion de la protéine à la membrane interne de la mitochondrie. Cependant, aucun effet de ces trois mutations n'a pu être mis en évidence sur l'efficacité ou la maturation de la protéine MR-1 (Ghezzi et al., 2009) soulevant la question d'une fonction différente, propre à ce peptide signal. Bien que très attractive, l'hypothèse d'une activité similaire à la glyoxylase II pour le gène MR-1 reste donc à confirmer.

Les trois mutations décrites dans ce gène sont localisées dans l'exon 1 qui est également commun avec la forme MR-1S, forme ubiquitairement exprimée dans les tissus périphériques et le cerveau (Lee et al., 2004). L'inhibition de l'expression de cette isoforme par RNA interférence dans des cellules Hep G2 (lignée de carcinome hépatique humain) a montré une inhibition de la prolifération cellulaire, de la migration et de l'adhésion (Ren et al., 2008). Cette isoforme pourrait donc, comme dans le cas du gène TAF1, être responsable de l'apparition de la maladie lorsqu'elle serait porteuse de mutations via la régulation d'autres gènes. Bien qu'aucun lien avec la voie de la dopamine n'ait été jusqu'ici mis en évidence dans cette forme de dystonie, il serait intéressant de comparer le niveau d'expression du gène $D R D 2$ en fonction $d u$ niveau d'expression du gène MR-1 chez ces patients afin d'explorer la possibilité de ce lien. 
Grâce à l'étude d'une grande famille, un second gène a été impliqué dans le DPNK et a pu être localisé sur le chromosome 2 (forme DYT20) (Spacey et al., 2006). L'identification de ce gène pourrait permettre de mieux comprendre la fonction de MR-1 ainsi que le mécanisme pathogène impliqué dans ces formes de dystonies.

\section{DYT12 et DYT16 : dystonies- parkinsonismes résultant d'une réponse inappropriée au stress?}

La forme DYT12 est une forme de dystonie-parkinsonisme autosomique dominante qui débute brutalement à l'adolescence ou chez le jeune adulte (Brashear et al., 1997). En quelques heures à quelques jours, parfois au décours d'un stress physique ou psychologique, s'installe un tableau neurologique sévère associant postures dystoniques des membres avec un gradient rostro-caudal, une bradykinésie, une perte des réflexes de posture ainsi que des signes d'atteinte bulbaire. Le tableau clinique reste ensuite relativement stable et les traitements médicaux, y compris la dopathérapie, sont peu efficaces. Cette forme de dystonie est liée à une mutation dans le gène ATP1A3 qui code pour une sous-unité spécifique du cerveau d'une pompe $\mathrm{NA}^{+} / \mathrm{K}^{+}$(de Carvalho Aguiar et al., 2004). Il a été précédemment suggéré que cette pompe jouait un rôle clé dans la dépolarisation et la décharge répétée des potentiels d'action des neurones adultes (Blanco et Mercer, 1998). Du fait de sa faible affinité pour les cations et sa haute affinité pour l'ATP, cette isoforme pourrait être mise en jeu lorsque les autres pompes $\mathrm{Na}^{+} / \mathrm{K}^{+}$seraient déjà au maximum de leur activité, permettant un retour plus efficace du potentiel membranaire au niveau basal. Le fonctionnement réduit de cette pompe pourrait donc entraîner une réponse physiologique exacerbée au stress. L'expression de ce gène $a$, elle aussi, été démontrée sous le contrôle du facteur de transcription Sp1 (Benfante et al., 2005).

Plus récemment, des mutations portées par le gène protein kinase, interferon-inducible double-stranded RNA-dependent activator (PRKRA) ont été associées à une nouvelle forme DYT16 de dystonie-parkinsonisme précoce de transmission autosomique récessive (Camargos et al., 2008 ; Seibler et al., 2008). Le tableau clinique est celui d'une dystonie apparaissant vers 12 ans se généralisant progressivement, avec une atteinte importante de l'axe, une atteinte oromandibulaire, et des signes parkinsoniens. Les traitements, notamment la dopathérapie, sont très peu efficaces (Camargos et al., 2008).

La protéine PRKRA est un activateur sensible au stress de la PKR, une protéine kinase activée par les ARN doubles brins (ARNd) et inductible par l'interféron (Patel et Sen, 1998 ; Peters et al., 2001). La PKR, constitutivement exprimée, peut être activée par phosphorylation à la suite d'une infection virale, par l'interféron de types I et III via des séquences spécifiques présentes dans son promoteur (Ank et al., 2006), ou par la PRKRA via des interactions protéine-protéine directes (Li et al., 2006). Cette activation de la PKR est suivie par une dimérisation qui permet la formation de la forme active de l'enzyme. Elle peut ainsi phosphoryler EIF2 qui va ainsi séquestrer le GDP. La cellule est ainsi mise en mode " pause " pour la traduction, permettant une reconfiguration, par d'autres voies de régulation, de l'expression des gènes dans la cellule en fonction des nouvelles conditions. La PRKRA est aussi un composant du complexe Dicer qui permet la régulation de la synthèse protéique via la destruction par clivage des ARNs messagers (Lee et al., 2006). La régulation de l'expression basale de la PRKRA, ainsi que de celle de la PKR, par le facteur de transcription Sp1 a également été rapportée (Fasciano et al., 2007). Aucune information supplémentaire n'est apportée par la localisation des mutations dans la protéine. En effet, la première mutation décrite, retrouvée homozygote dans deux familles (c.665C > T, p.Pro222Leu) (Camargos et al., 2008), n'est pas située dans les domaines 1 et 2 d'interaction avec la PKR, ni dans le domaine 3 permettant l'activation de cette dernière (Patel et Sen, 1998 ; Peters et al., 2001). La seconde mutation, retrouvée de façon hétérozygote chez un patient, est située dans le premier domaine (c.266_267delAT, p.His89fsX20) (Seibler et al., 2008), mais entraîne l'apparition d'un codon stop prématuré et donc d'une protéine tronquée. En accord avec un mode de transmission autosomique récessif, il pourrait s'agir de la première mutation identifiée chez un hétérozygote composite (la seconde mutation étant localisée sur un second gène non encore identifié).

Les anomalies du gène PRKRA rapportées dans les formes DYT16 pourraient donc, d'une part, empêcher la reconfiguration de l'expression des gènes en conditions de stress et, d'autre part, pourraient également participer à la régulation de la traduction par destruction des ARNm.

\section{DYT6 et THAP1 : un lien avec la dopamine ?}

DYT6 a été décrite, comme pour la dystonie de Lubag, dans un isolat génétique : les Amish-Mennonites (Almasy et al., 1997 ; Saunders-Pullman et al., 2007). Une mutation fondatrice dans le gène THAP1 a été rapportée dans quatre familles de cette population (mutation c.134_135insGGGTT ;137_139delAAC qui correspond à une insertion/délétion dans l'exon 2 provoquant un décalage du cadre de lecture et un codon stop prématuré) (Fuchs et al., 2009). La caractérisation d'une mutation fauxsens (c.241T > C ; p.Phe81Leu) dans une cinquième famille pour laquelle aucune origine mennonite n'a été retrouvée avait entrouvert la possibilité de l'implication de mutations de ce gène chez des patients DYT6 appartenant à d'autres populations (Fuchs et al., 2009). Cela a été récemment confirmé par l'identification de mutations dans 11 familles (Bressman et al., 2009 ; Djarmati et al., 2009). La dystonie DYT6 apparaît généralement dans la seconde décade, mais l'éventail de l'âge de début est large (deux à 49 ans). Il s'agit d'une dystonie mixte, impliquant les membres supérieurs, mais surtout la région craniocéphalique, cette atteinte oromandibulaire et cervicale étant la principale responsable du handicap fonctionnel. Une généralisation est possible (Bressman et al., 2009 ; Djarmati et al., 2009).

THAP1 est un facteur nucléaire défini par un domaine " THAP " particulier de liaison à l'ADN en doigt de zinc. Il est retrouvé chez des animaux modèles invertébrés comme la drosophile, le nématode, mais aussi vertébrés comme le zébrafish, traduisant une forte pression de sélection sur ce domaine et laissant soupçonner le rôle clé des gènes qu'il régule (Clouaire et al., 2005). Il a d'abord été montré comme 
étant un facteur sensibilisant les cellules à l'apoptose et interagissant avec prostate-apoptosis-response-4 (Par-4) (Roussigne et al., 2003), une protéine décrite comme co-facteur transcriptionnel (Johnstone et al., 1996; Richard et al., 2001) et impliquée dans le cancer de la prostate et certaines maladies neurodégénératives comme la maladie de Parkinson (Duan et al., 1999). THAP1 pourrait ainsi recruter Par-4 à des sites spécifiques afin de favoriser/défavoriser l'expression de gènes cibles. Des études visant à réduire l'expression de THAP1 par ARN interférence montre une inhibition de la prolifération cellulaire, de la transition de la phase G1 à la phase $\mathrm{S}$ du cycle cellulaire ainsi qu'une régulation négative de la voie $\mathrm{pRB} / \mathrm{E} 2 \mathrm{~F}$ (Cayrol et al., 2007). Curieusement, la surexpression de cette protéine entraîne également une inhibition de la prolifération. L'action de THAP1 semble donc être déterminée par l'adéquation de son expression à une gamme quantitative très précise.

Bien que le lien entre dopamine et THAP1 soit encore inconnu, des résultats d'imagerie cérébrale visualisant l'activité des récepteurs dopaminergiques $D_{2}$ démontrent une baisse significative $(-38,0 \pm 3 \%)$ de la disponibilité de ce récepteur dans le striatum d'une cohorte de patients DYT6 (mutés pour le gène THAP1) lorsqu'il est comparé au striatum de contrôles sains (Carbon et Eidelberg, 2009).

\section{Conclusion}

Les différents gènes qui ont été identifiés chez des patients atteints de dystonie interviennent donc dans l'homéostasie du neurone en réponse à des changements de conditions du milieu. Ces gènes vont induire la synthèse d'autres gènes et la traduction de protéines, permettant la mise en place de mécanismes de défense et de réparation pouvant se traduire par un remaniement membranaire, la récupération d'un équilibre ionique ou la destruction de produits toxiques. Les différentes formes de dystonies pourraient donc être dues à une perte d'efficacité du neurone et/ou son incapacité à revenir à un état de fonctionnement basal. Les gènes impliqués dans ces différentes formes de dystonies appartiennent donc à des mécanismes de régulation distincts qui peuvent plus ou moins être intégrés dans une voie de régulation complexe, mais unique, centrée sur la dopamine.

Du point de vue pratique, notamment thérapeutique, les données récentes de la génétique ne remettent pas en cause l'attitude qui consiste devant toute dystonie, notamment chez l'enfant, à introduire en première intention un traitement par L-dopa bien mené (Jankovic, 2006). Il s'agit là d'un test thérapeutique et non d'un test diagnostique (les DRD étant diagnostiquées par l'analyse du liquide céphalorachidien et d'autres formes de dystonies primaires pouvant rarement certes, mais parfois, être améliorées par la dopathérapie).

Enfin, le schéma consistant à associer un seul gène à chacune des différentes formes de dystonies est, bien entendu, simplificateur. La pénétrance incomplète observée dans de nombreuses formes (i.e. la présence de personnes porteuses de la mutation, mais ne développant pas la maladie) ainsi que la variabilité dans la sévérité des formes cliniques observées pour une même mutation montre qu'il faut très probablement se référer à un modèle de transmission plus complexe faisant intervenir un gène majeur, il est vrai, mais également des gènes modificateurs ainsi que des facteurs environnementaux. Seule la partie visible de l'iceberg est actuellement prospectée et il est très probable que, dans le futur, de nombreux acteurs seront mis à contribution pour rendre compte de toute la complexité de ces maladies multifactorielles.

\section{Conflit d'intérêt}

Les auteurs ne déclarent aucun conflit d'intérêt.

\section{RÉ F ÉRENCES}

Almasy L, Bressman S, Raymond D, Kramer P, Greene P, Heiman $\mathrm{G}$, et al. Idiopathic torsion dystonia linked to chromosome 8 in two Mennonite families. Ann Neurol 1997;42:670-3.

Angelini C, Fanin M, Freda MP, Duggan DJ, Siciliano G, Hoffman EP. The clinical spectrum of sarcoglycanopathies. Neurology 1999;52:176-9.

Ank N, West H, Bartholdy C, Eriksson K, Thomsen AR, Paludan SR. Lambda interferon (IFN-lambda), a type III IFN, is induced by viruses and IFNs and displays potent antiviral activity against select virus infections in vivo. J Virol 2006;80:4501-9.

Aosaki T, Kiuchi K, Kawaguchi Y. Dopamine D1-like receptor activation excites rat striatal large aspiny neurons in vitro. J Neurosci 1998;18:5180-90.

Asanuma K, Ma Y, Okulski J, Dhawan V, Chaly T, Carbon M, et al. Decreased striatal D2 receptor binding in nonmanifesting carriers of the DYT1 dystonia mutation. Neurology 2005;64:347-9.

Augood SJ, Hollingsworth Z, Albers DS, Yang L, Leung JC, Muller B, et al. Dopamine transmission in DYT1 dystonia: a biochemical and autoradiographical study. Neurology 2002;59:445-8.

Augood SJ, Martin DM, Ozelius LJ, Breakefield XO, Penney Jr JB, Standaert DG. Distribution of the mRNAs encoding torsin $A$ and torsinB in the normal adult human brain. Ann Neurol 1999;46:761-9.

Benfante R, Antonini RA, Vaccari M, Flora A, Chen F, Clementi F, et al. The expression of the human neuronal alpha3 $\mathrm{Na}^{+}, \mathrm{K}^{+}-$ ATPase subunit gene is regulated by the activity of the Sp1 and NF-Y transcription factors. Biochem J 2005; 386:63-72.

Beukers RJ, Booij J, Weisscher N, Zijlstra F, van Amelsvoort TA, Tijssen MA. Reduced striatal D2 receptor binding in myoclonus-dystonia. Eur J Nucl Med Mol Imaging 2009;36:269-74.

Blake DJ, Weir A, Newey SE, Davies KE. Function and genetics of dystrophin and dystrophin-related proteins in muscle. Physiol Rev 2002;82:291-329.

Blanco G, Mercer RW. Isozymes of the Na-K-ATPase: heterogeneity in structure, diversity in function. Am J Physiol 1998;275:F633-50.

Brashear A, DeLeon D, Bressman SB, Thyagarajan D, Farlow MR, Dobyns WB. Rapid-onset dystonia-parkinsonism in a second family. Neurology 1997;48:1066-9.

Breakefield XO, Blood AJ, Li Y, Hallett M, Hanson PI, Standaert DG. The pathophysiological basis of dystonias. Nat Rev Neurosci 2008;9:222-34.

Bressman SB, Sabatti C, Raymond D, de Leon D, Klein C, Kramer $\mathrm{PL}$, et al. The DYT1 phenotype and guidelines for diagnostic testing. Neurology 2000;54:1746-52. 
Bressman SB, Raymond D, Fuchs T, Heiman GA, Ozelius LJ, Saunders-Pullman R. Mutations in THAP1 (DYT6) in earlyonset dystonia: a genetic screening study. Lancet Neurol 2009;8:441-6.

Cabrera-Vera TM, Hernandez S, Earls LR, Medkova M, SundgrenAndersson AK, Surmeier DJ, et al. RGS9-2 modulates D2 dopamine receptor-mediated $\mathrm{Ca}^{2+}$ channel inhibition in rat striatal cholinergic interneurons. Proc Natl Acad Sci U S A 2004;101:16339-44.

Calabresi P, Centonze D, Pisani A, Sancesario G, North RA Bernardi G. Muscarinic IPSPs in rat striatal cholinergic interneurones. J Physiol 1998;510:421-7.

Camargos S, Scholz S, Simon-Sanchez J, Paisan-Ruiz C, Lewis P, Hernandez D, et al. DYT16, a novel young-onset dystoniaparkinsonism disorder: identification of a segregating mutation in the stress-response protein PRKRA. Lancet Neurol 2008;7:207-15.

Cao S, Gelwix CC, Caldwell KA, Caldwell GA. Torsin-mediated protection from cellular stress in the dopaminergic neurons of Caenorhabditis elegans. J Neurosci 2005;25:3801-12.

Carbon M, Eidelberg D. Abnormal structure-function relationships in hereditary dystonia. Neuroscience 2009. doi: 10.1016/j.neuroscience.2009.12.041 [Epub ahead of Print].

Cayrol C, Lacroix C, Mathe C, Ecochard V, Ceribelli M, Loreau E, et al. The THAP-zinc finger protein THAP1 regulates endothelial cell proliferation through modulation of $\mathrm{pRB} /$ E2F cell-cycle target genes. Blood 2007;109:584-94.

Chan P, Gonzalez-Maeso J, Ruf F, Bishop DF, Hof PR, Sealfon SC. Epsilon-sarcoglycan immunoreactivity and mRNA expression in mouse brain. J Comp Neurol 2005;482:50-73.

Chouery E, Kfoury J, Delague V, Jalkh N, Bejjani P, Serre JL, et al. A novel locus for autosomal recessive primary torsion dystonia (DYT17) maps to 20p11.22-q13.12. Neurogenetics 2008;9:287-93.

Clot F, Grabli D, Cazeneuve C, Roze E, Castelnau P, Chabrol B, et al. The French Dystonia Network, 2009. Exhaustive analysis of $\mathrm{BH} 4$ and dopamine biosynthesis genes in patients with Dopa-responsive dystonia. Brain 2009;132:1753-63.

Clouaire T, Roussigne M, Ecochard V, Mathe C, Amalric F, Girard JP. The THAP domain of THAP1 is a large C2CH module with zinc-dependent sequence-specific DNA-binding activity. Proc Natl Acad Sci U S A 2005;102:6907-12.

Cohen G. Oxy-radical toxicity in catecholamine neurons. Neurotoxicology 1984;5:77-82.

Dauer W, Przedborski S. Parkinson's disease: mechanisms and models. Neuron 2003;39:889-909.

de Carvalho Aguiar P, Sweadner KJ, Penniston JT, Zaremba J, Liu $\mathrm{L}$, Caton $\mathrm{M}$, et al. Mutations in the $\mathrm{Na}+\mathrm{K}+$-ATPase alpha3 gene ATP1A3 are associated with rapid-onset dystonia parkinsonism. Neuron 2004;43:169-75.

Deng P, Zhang Y, Xu ZC. Involvement of I(h) in dopamine modulation of tonic firing in striatal cholinergic interneurons. J Neurosci 2007;27:3148-56.

Djarmati A, Schneider SA, Lohmann K, Winkler S, Pawlack H, Hagenah J, et al. Mutations in THAP1 (DYT6) and generalised dystonia with prominent spasmodic dysphonia: a genetic screening study. Lancet Neurol 2009;8:447-52.

Duan W, Zhang Z, Gash DM, Mattson MP. Participation of prostate apoptosis response- 4 in degeneration of dopaminergic neurons in models of Parkinson's disease. Ann Neurol 1999;46:587-97.

Dunah AW, Jeong H, Griffin A, Kim YM, Standaert DG, Hersch $\mathrm{SM}$, et al. Sp1 and TAFII130 transcriptional activity disrupted in early Huntington's disease. Science 2002;296:2238-43.

Esapa CT, Waite A, Locke M, Benson MA, Kraus M, Mcllhinney RA, et al. SGCE missense mutations that cause myoclonus-dystonia syndrome impair epsilon-sarcoglycan trafficking to the plasma membrane: modulation by ubiquitination and torsinA. Hum Mol Genet 2007;16:327-42.

Fasciano S, Kaufman A, Patel RC. Expression of PACT is regulated by $\mathrm{Sp} 1$ transcription factor. Gene 2007;388:74-82.

Frédéric MY, Clot F, Cif L, Blanchard A, Dürr A, Vuillaume I, et al Is the early-onset torsion dystonia (EOTD) linked to TOR1A gene as frequent as expected in France? Neurogenetics 2008;9:143-50.

Friedman J, Hyland K, Blau N, MacCollin M. Dopa-responsive hypersomnia and mixed movement disorder due to sepiapterin reductase deficiency. Neurology 2006;67:2032-5.

Fuchs T, Gavarini S, Saunders-Pullman R, Raymond D, Ehrlich ME, Bressman SB, et al. Mutations in the THAP1 gene are responsible for DYT6 primary torsion dystonia. Nat Genet 2009;41:286-8.

Furukawa Y, Hornykiewicz O, Fahn S, Kish SJ. Striatal dopamine in early-onset primary torsion dystonia with the DYT1 mutation. Neurology 2000;54:1193-5.

Furukawa Y. Update on dopa-responsive dystonia: locus heterogeneity and biochemical features. Adv Neurol 2004:94:127-38.

Gavarini S, Vayssière N, Delort P, Cif L, Biolsi B, Tancu C, et al. Stereotactic MRI in DYT1 dystonia: focal signal abnormalities in the basal ganglia do not contraindicate deep brain stimulation. Stereotact Funct Neurosurg 2008;86:245-52.

Geyer HL, Bressman SB. The diagnosis of dystonia. Lancet Neurol 2006;5:780-90

Ghezzi D, Viscomi C, Ferlini A, Gualandi F, Mereghetti P, De Grandis D, et al. Paroxysmal non-kinesigenic dyskinesia is caused by mutations of the MR-1 mitochondrial targeting sequence. Hum Mol Genet 2009;18:1058-64.

Goodchild RE, Kim CE, Dauer WT. Loss of the dystoniaassociated protein torsinA selectively disrupts the neuronal nuclear envelope. Neuron 2005;48:923-32.

Goto S, Lee LV, Munoz EL, Tooyama I, Tamiya G, Makino S, et al. Functional anatomy of the basal ganglia in X-linked recessive dystonia-parkinsonism. Ann Neurol 2005;58:7-17.

Grabowski M, Zimprich A, Lorenz-Depiereux B, Kalscheuer V, Asmus F, Gasser T, et al. The epsilon-sarcoglycan gene (SGCE), mutated in myoclonus-dystonia syndrome, is maternally imprinted. Eur J Hum Genet 2003;11:138-44.

Granata A, Watson R, Collinson LM, Schiavo G, Warner TT. The dystonia-associated protein torsinA modulates synaptic vesicle recycling. J Biol Chem 2008;283:7568-79.

Graybiel AM, Canales JJ, Capper-Loup C. Levodopa-induced dyskinesias and dopamine-dependent stereotypies: a new hypothesis. Trends Neurosci 2000;23:S71-7.

Grotzsch H, Pizzolato GP, Ghika J, Schorderet D, Vingerhoets FJ, Landis T, et al. Neuropathology of a case of dopa-responsive dystonia associated with a new genetic locus, DYT14. Neurology 2002;58:1839-42.

Hess CW, Raymond D, Aguiar Pde C, Frucht S, Shriberg J, Heiman GA, et al. Myoclonus-dystonia, obsessivecompulsive disorder, and alcohol dependence in SGCE mutation carriers. Neurology 2007;68:522-4.

Hewett JW, Tannous B, Niland BP, Nery FC, Zeng J, Li Y, et al. Mutant torsinA interferes with protein processing through the secretory pathway in DYT1 dystonia cells. Proc Natl Acad Sci U S A 2007;104:7271-6.

Hisatake K, Hasegawa S, Takada R, Nakatani Y, Horikoshi M, Roeder RG. The p250 subunit of native TATA box-binding factor TFIID is the cell-cycle regulatory protein CCG1. Nature 1993;362:179-81.

Horie C, Suzuki Y, Kiyosawa M, Mochizuki M, Wakakura M, Oda $\mathrm{K}$, et al. Decreased dopamine D-2 receptor binding in essential blepharospasm. Acta Neurologica Scand 2009;119:49-54 
Hyland K, Gunasekara RS, Munk-Martin TL, Arnold LA, Engle T. The hph-1 mouse: a model for dominantly inherited GTPcyclohydrolase deficiency. Ann Neurol 2003;54(Suppl. 6):S46-8.

Ichinose H, Ohye T, Takahashi E, Seki N, Hori T, Segawa M, et al. Hereditary progressive dystonia with marked diurnal fluctuation caused by mutations in the GTP cyclohydrolase I gene. Nat Genet 1994;8:236-42.

Jankovic J. Treatment of dystonia. Lancet Neurol 2006;5:864-72.

Johnstone RW, See RH, Sells SF, Wang J, Muthukkumar S, Englert C, et al. A novel repressor, Par-4, modulates transcription and growth suppression functions of the Wilms' tumor suppressor WT1. Mol Cell Biol 1996;16:6945-56.

Kupke KG, Lee LV, Viterbo GH, Arancillo J, Donlon T, Muller U. Xlinked recessive torsion dystonia in the Philippines. Am J Med Genet 1990;36:237-42.

Lee HY, Xu Y, Huang Y, Ahn AH, Auburger GW, Pandolfo M, et al. The gene for paroxysmal non-kinesigenic dyskinesia encodes an enzyme in a stress response pathway. Hum Mol Genet 2004;13:3161-70.

Lee V, Pascasio F, Fuentes F, Viterbo G. Torsion dystonia in Panay Philippines. Adv Neurol 1976;14:137-51.

Lee Y, Hur I, Park SY, Kim YK, Suh MR, Kim VN. The role of PACT in the RNA silencing pathway. EMBO J 2006;25:522-32.

Li S, Peters GA, Ding K, Zhang X, Qin J, Sen GC. Molecular basis for PKR activation by PACT or dsRNA. Proc Natl Acad Sci U S A 2006;103:10005-10.

Li TB, Liu XH, Feng S, Hu Y, Yang WX, Han Y, et al. Characterization of MR-1, a novel myofibrillogenesis regulator in human muscle. Acta Biochim Biophys Sin (Shanghai) 2004;36:412-8.

Luciano MS, Ozelius L, Sims K, Raymond D, Liu L, SaundersPullman R. Responsiveness to levodopa in epsilonsarcoglycan deletions. Mov Disord 2009;24:425-8.

Lüdecke B, Dworniczak B, Bartholomé K. A point mutation in the tyrosine hydroxylase gene associated with Segawa's syndrome. Hum Genet 1995;95:123-5.

Makino S, Kaji R, Ando S, Tomizawa M, Yasuno K, Goto S, et al. Reduced neuron-specific expression of the TAF1 gene is associated with X-linked dystonia-parkinsonism. Am J Hum Genet 2007;80:393-406.

Maréchal L, Raux G, Dumanchin C, Lefebvre G, Deslandre E, Girard C, et al. Severe myoclonus-dystonia syndrome associated with a novel epsilon-sarcoglycan gene truncating mutation. Am J Med Genet B Neuropsychiatr Genet 2003;119B:114-7.

Morange M. Protéines chaperons. Med Sci 2000;16:630-4.

Naismith TV, Heuser JE, Breakefield XO, Hanson PI. TorsinA in the nuclear envelope. Proc Natl Acad Sci U S A 2004:101:7612-7.

Nishiyama A, Endo T, Takeda S, Imamura M. Identification and characterization of epsilon-sarcoglycans in the central nervous system. Brain Res Mol Brain Res 2004;125:1-12.

Ozelius L, Hewett J, Page C, Bressman S, Kramer P, Shalish C, et al. The early-onset torsion dystonia gene (DYT1) encodes an ATP-binding protein. Nat Genet 1997;17:40-8.

Patel RC, Sen GC. PACT, a protein activator of the interferoninduced protein kinase, PKR. EMBO J 1998;17:4379-90.

Peters GA, Hartmann R, Qin J, Sen GC. Modular structure of PACT: distinct domains for binding and activating PKR. Mol Cell Biol 2001;21:1908-20.

Perlmutter JS, Stambuk MK, Markham J, Black KJ, McGeeMinnich L, Jankovic J, et al. Decreased [F18]spiperone binding in putamen in idiopathic focal dystonia. J Neurosci 1997;17:843-50.

Perlmutter JS, Mink JW. Dysfunction of dopaminergic pathways in dystonia. Adv Neurol 2004;94:163-70.
Pisani A, Bernardi G, Ding J, Surmeier DJ. Re-emergence of striatal cholinergic interneurons in movement disorders. Trends Neurosci 2007:30:545-53.

Pisani A, Martella G, Tscherter A, Bonsi P, Sharma N, Bernardi G, et al. Altered responses to dopaminergic D2 receptor activation and $\mathrm{N}$-type calcium currents in striatal cholinergic interneurons in a mouse model of DYT1 dystonia. Neurobiol Dis 2006;24:318-25.

Pons R. The phenotypic spectrum of paediatric neurotransmitter diseases and infantile parkinsonism. J Inherit Metab Dis 2009;32:321-32.

Rainier S, Thomas D, Tokarz D, Ming L, Bui M, Plein E, et al. Myofibrillogenesis regulator 1 gene mutations cause paroxysmal dystonic choreoathetosis. Arch Neurol 2004;61:1025-9.

Ren $\mathrm{K}$, Jin H, Bian C, He H, Liu X, Zhang S, et al. MR-1 modulates proliferation and migration of human hepatoma HepG2 cells through myosin light chains-2 (MLC2)/focal adhesion kinase (FAK)/Akt signaling pathway. J Biol Chem 2008;283:35598-605.

Richard DJ, Schumacher V, Royer-Pokora B, Roberts SG. Par4 is a coactivator for a splice isoform-specific transcriptional activation domain in WT1. Genes Dev 2001;15:328-39.

Rinne JO, Iivanainen M, Metsähonkala L, Vainionpää L, Pääkkönen L, Någren K, et al. Striatal dopaminergic system in dopa-responsive dystonia: a multi-tracer PET study shows increased D2 receptors. J Neural Transm 2004; 111:59-67.

Rostasy K, Augood SJ, Hewett JW, Leung JC, Sasaki H, Ozelius LJ, et al. TorsinA protein and neuropathology in early onset generalized dystonia with GAG deletion. Neurobiol Dis 2003;12:11-24.

Roussigne M, Cayrol C, Clouaire T, Amalric F, Girard JP. THAP1 is a nuclear proapoptotic factor that links prostate-apoptosisresponse-4 (Par-4) to PML nuclear bodies. Oncogene 2003;22:2432-42.

Saunders-Pullman R, Raymond D, Senthil G, Kramer P, Ohmann E, Deligtisch A, et al. Narrowing the DYT6 dystonia region and evidence for locus heterogeneity in the AmishMennonites. Am J Med Genet A 2007;143A:2098-105.

Sciamanna G, Bonsi P, Tassone A, Cuomo D, Tscherter A, Viscomi MT, et al. Impaired striatal D2 receptor function leads to enhanced GABA transmission in a mouse model of DYT1 dystonia. Neurobiol Dis 2009;34:133-45.

Seibler P, Djarmati A, Langpap B, Hagenah J, Schmidt A, Bruggemann $\mathrm{N}$, et al. A heterozygous frameshift mutation in PRKRA (DYT16) associated with generalised dystonia in a German patient. Lancet Neurol 2008;7:380-1.

Sharma N, Baxter MG, Petravicz J, Bragg DC, Schienda A, Standaert DG, et al. Impaired motor learning in mice expressing torsinA with the DYT1 dystonia mutation. J Neurosci 2005;25:5351-5.

Shashidharan P, Kramer BC, Walker RH, Olanow CW, Brin MF. Immunohistochemical localization and distribution of torsinA in normal human and rat brain. Brain Res 2000;853:197-206.

Shiga K, Yoshioka H, Matsumiya T, Kimura I, Takeda S, Imamura M. Zeta-sarcoglycan is a functional homologue of gamma-sarcoglycan in the formation of the sarcoglycan complex. Exp Cell Res 2006;312:2083-92.

Spacey SD, Adams PJ, Lam PC, Materek LA, Stoessl AJ, Snutch $\mathrm{TP}$, et al. Genetic heterogeneity in paroxysmal nonkinesigenic dyskinesia. Neurology 2006;66:1588-90.

Torres GE, Sweeney AL, Beaulieu JM, Shashidharan P, Caron MG. Effect of torsinA on membrane proteins reveals a loss of function and a dominant-negative phenotype of the dystonia-associated DeltaE-torsinA mutant. Proc Natl Acad Sci U S A 2004;101:15650-5. 
Valente EM, Misbahuddin A, Brancati F, Placzek MR, Garavaglia $\mathrm{B}$, Salvi S, et al. Analysis of the epsilon-sarcoglycan gene in familial and sporadic myoclonus-dystonia: evidence for genetic heterogeneity. Mov Disord 2003;18:1047-51.

Vidailhet M, Dupel C, Lehéricy S, Remy P, Dormont D, Serdaru $\mathrm{M}$, et al. Dopaminergic dysfunction in midbrain dystonia: anatomoclinical study using 3-dimensional magnetic resonance imaging and fluorodopa $\mathrm{F} 18$ positron emission tomography. Arch Neurol 1999;56:982-9.

Vidailhet M, Grabli D, Roze E. Pathophysiology of dystonia. Curr Opin Neurol 2009;22(4):406-13.

Vitek JL. Pathophysiology of dystonia: a neuronal model. Mov Disord 2002;17(Suppl. 3):S49-62.

Waite A, Tinsley CL, Locke M, Blake DJ. The neurobiology of the dystrophin-associated glycoprotein complex. Ann Med 2009;41:344-59.

Weber YG, Storch A, Wuttke TV, Brockmann K, Kempfle J, Maljevic S, et al. GLUT1 mutations are a cause of paroxysmal exertion-induced dyskinesias and induce hemolytic anemia by a cation leak. J Clin Invest 2008;118:2157-68.

Wheeler MT, Zarnegar S, McNally EM. Zeta-sarcoglycan, a novel component of the sarcoglycan complex, is reduced in muscular dystrophy. Hum Mol Genet 2002;11:2147-54
Wider C, Melquist S, Hauf M, Solida A, Cobb SA, Kachergus JM, et al. Study of a Swiss dopa-responsive dystonia family with a deletion in GCH1: redefining DYT14 as DYT5. Neurology 2008;70:1377-83.

Wichmann T. Commentary: Dopaminergic dysfunction in DYT1 dystonia. Exp Neurol 2008;212:242-6.

Xiao J, LeDoux MS. Cloning, developmental regulation and neural localization of rat epsilon-sarcoglycan. Brain Res Mol Brain Res 2003;119:132-43.

Yokoi F, Dang MT, Li J, Li Y. Myoclonus, motor deficits, alterations in emotional responses and monoamine metabolism in epsilon-sarcoglycan deficient mice. J Biochem 2006;140:141-6.

Zanotti-Fregonara P, Vidailhet M, Kas A, Ozelius LJ, Clot F, Hindié E, et al. [ $\left.{ }^{123} \mathrm{I}\right]-\mathrm{FP}$-CIT and [99mTc]-HMPAO single photon emission computed tomography in a new sporadic case of rapid-onset dystonia-parkinsonism. J Neurol Sci 2008;273:148-51.

Zhou FM, Wilson CJ, Dani JA. Cholinergic interneuron characteristics and nicotinic properties in the striatum. J Neurobiol 2002;53:590-605.

Zimprich A, Grabowski M, Asmus F, Naumann M, Berg D, Bertram $\mathrm{M}$, et al. Mutations in the gene encoding epsilonsarcoglycan cause myoclonus-dystonia syndrome. Nat Genet 2001;29:66-9. 